\title{
Estimates for lower bounds of eigenvalues of the poly-Laplacian and quadratic polynomial operator of the Laplacian
}

\author{
Qing-Ming Cheng He-Jun Sun Guoxin Wei Lingzhong Zeng
}

\begin{abstract}
In this paper, we investigate the Dirchlet eigenvalue problems of polyLaplacian with any order and quadratic polynomial operator of the Laplacian. We give some estimates for lower bounds of the sums of their first $k$ eigenvalues which improve the previous results.
\end{abstract}

\section{Introduction}

Let $\Omega$ be a bounded domain in an $n$-dimensional Euclidean space $\mathbb{R}^{n}$, where $n \geq 2$. The Dirichlet eigenvalue problem of the poly-Laplacian is described by

$$
\left\{\begin{array}{l}
(-\Delta)^{l} u=\lambda u, \quad \text { on } \Omega, \\
\left.u\right|_{\partial \Omega}=\left.\frac{\partial u}{\partial \nu}\right|_{\partial \Omega}=\cdots=\left.\frac{\partial^{l-1} u}{\partial \nu^{l-1}}\right|_{\partial \Omega}=0,
\end{array}\right.
$$

where $\Delta$ is the Laplacian and $\nu$ denotes the outward unit normal vector field of $\partial \Omega$. As we known, this problem has a real and discrete spectrum: $0<\lambda_{1} \leq \lambda_{2} \leq \cdots \leq$ $\lambda_{k} \leq \cdots \rightarrow \infty$, where each eigenvalue repeats with its multiplicity.

When $l=1$, problem (1.1) is called the Dirichlet Laplacian problem or the fixed membrane problem. The asymptotic behavior of its $k$-th eigenvalue $\lambda_{k}$ relates to geometric properties of $\Omega$ when $k \rightarrow \infty$. In fact, the following Weyl's asymptotic formula holds

$$
\lambda_{k} \sim \frac{(2 \pi)^{2}}{\left(\omega_{n} V(\Omega)\right)^{\frac{2}{n}}} k^{\frac{2}{n}}, \quad \text { as } k \rightarrow \infty
$$

where $\omega_{n}$ denotes the volume of the unit ball in $\mathbb{R}^{n}$ and $V(\Omega)$ denotes the volume of $\Omega$. In 1961, Pólya [13] proved that

$$
\lambda_{k} \geq \frac{(2 \pi)^{2}}{\left(\omega_{n} V(\Omega)\right)^{\frac{2}{n}}} k^{\frac{2}{n}}
$$

2010 Mathematics Subject Classification: 35P15.

Key words and phrases: eigenvalue, poly-Laplacian, quadratic polynomial operator of the Laplacian.

The first author was supported by a Grant-in-Aid for Scientific Research from JSPS. The second author and the third author were supported by the National Natural Science Foundation of China (Grant Nos.11001130, 11001087). 
holds on tiling domains in $\mathbb{R}^{2}$. His proof also works on tiling domains in $\mathbb{R}^{n}$. Moreover, he conjectured that (1.3) holds for any bounded domain in $\mathbb{R}^{n}$. Berezin [3] and Lieb [10] made some contributions to the partial solution of this conjecture. In 1983, Li and Yau [9] proved the following so-called Li-Yau inequality

$$
\frac{1}{k} \sum_{j=1}^{k} \lambda_{j} \geq \frac{n}{n+2} \frac{(2 \pi)^{2}}{\left(\omega_{n} V(\Omega)\right)^{\frac{2}{n}}} k^{\frac{2}{n}}
$$

In 2000, Laptev and Weidl [7] pointed out that (1.4) can be derived by the Legendre transform of a result derived by Berezin [3]. Hence, (1.4) is also called the BerezinLi-Yau inequality. In 2003, adding an additional positive term to the right-hand side of (1.4), Melas [1] improved (1.4) to

$$
\frac{1}{k} \sum_{j=1}^{k} \lambda_{j} \geq \frac{n}{n+2} \frac{(2 \pi)^{2}}{\left(\omega_{n} V(\Omega)\right)^{\frac{2}{n}}} k^{\frac{2}{n}}+\frac{1}{24(n+2)} \frac{V(\Omega)}{I(\Omega)},
$$

where $I(\Omega)=\min _{a \in \mathbb{R}^{n}} \int_{\Omega}|x-a|^{2} d x$ is the moment of inertia of $\Omega$. Recently, Ilyin [6] obtained the following asymptotic lower bound for eigenvalues of problem (1.1):

$$
\frac{1}{k} \sum_{j=1}^{k} \lambda_{j} \geq \frac{n}{n+2} \frac{(2 \pi)^{2}}{\left(\omega_{n} V(\Omega)\right)^{\frac{2}{n}}} k^{\frac{2}{n}}+\frac{n}{48} \frac{V(\Omega)}{I(\Omega)}\left(1-\varepsilon_{n}(k)\right),
$$

where $0 \leq \varepsilon_{n}(k)=O\left(k^{-\frac{2}{n}}\right)$ is a infinitesimal of $k^{-\frac{2}{n}}$. Moreover, he derived some explicit inequalities for the particular cases of $n=2,3,4$ :

$$
\frac{1}{k} \sum_{j=1}^{k} \lambda_{j} \geq \frac{n}{n+2} \frac{(2 \pi)^{2}}{\left(\omega_{n} V(\Omega)\right)^{\frac{2}{n}}} k^{\frac{2}{n}}+\frac{n}{48} \beta_{n} \frac{V(\Omega)}{I(\Omega)},
$$

where $\beta_{2}=\frac{119}{120}, \beta_{3}=0.986$ and $\beta_{4}=0.983$.

When $l=2$, problem (1.1) is called the clamped plate problem. Agmon [1] and Pleijel [12] obtained

$$
\lambda_{k} \sim \frac{(2 \pi)^{4}}{\left(\omega_{n} V(\Omega)\right)^{\frac{4}{n}}} k^{\frac{4}{n}}, \quad \text { as } k \rightarrow+\infty
$$

In 1985, Levine and Protter [8] proved:

$$
\frac{1}{k} \sum_{j=1}^{k} \lambda_{j} \geq \frac{n}{n+4} \frac{(2 \pi)^{4}}{\left(\omega_{n} V(\Omega)\right)^{\frac{4}{n}}} k^{\frac{4}{n}}
$$

For the special case of $n=2$, Ilyin [6] proved

$$
\frac{1}{k} \sum_{j=1}^{k} \lambda_{j} \geq \frac{16 \pi^{2}}{3(V(\Omega))^{2}} k^{2}+\frac{12095 \pi}{3 \cdot 12096 I(\Omega)} k .
$$


In 2011, Cheng and Wei [5] strengthened (1.9) to

$$
\begin{aligned}
\frac{1}{k} \sum_{j=1}^{k} \lambda_{j} \geq & \frac{n}{n+4} \frac{(2 \pi)^{4}}{\left(\omega_{n} V(\Omega)\right)^{\frac{4}{n}}} k^{\frac{4}{n}} \\
& +\frac{n}{n+2}\left[\frac{n+2}{12 n(n+4)}-\frac{1}{1152 n^{2}(n+4)}\right] \frac{(2 \pi)^{2}}{\left(\omega_{n} V(\Omega)\right)^{\frac{2}{n}}} \frac{V(\Omega)}{I(\Omega)} k^{\frac{2}{n}} \\
& +\left[\frac{1}{576 n(n+4)}-\frac{1}{27648 n^{2}(n+2)(n+4)}\right]\left(\frac{V(\Omega)}{I(\Omega)}\right)^{2} .
\end{aligned}
$$

When $l \geq 3$, Levine and Protter [8] proved

$$
\frac{1}{k} \sum_{j=1}^{k} \lambda_{j} \geq \frac{n}{n+2 l} \frac{(2 \pi)^{2 l}}{\left(\omega_{n} V(\Omega)\right)^{\frac{2 l}{n}}} k^{\frac{2 l}{n}} .
$$

Recently, adding $l$ terms of lower order of $k^{\frac{2 l}{n}}$ to its right-hand side of (1.12), Cheng, Qi and Wei [4] derived

$$
\begin{aligned}
\frac{1}{k} \sum_{j=1}^{k} \lambda_{j} & \geq \frac{n}{n+2 l} \frac{(2 \pi)^{2 l}}{\left(\omega_{n} V(\Omega)\right)^{\frac{2 l}{n}}} k^{\frac{2 l}{n}} \\
& +\frac{n}{(n+2 l)} \sum_{p=1}^{l} \frac{l+1-p}{(24)^{p} n \cdots(n+2 p-2)} \frac{(2 \pi)^{2(l-p)}}{\left(\omega_{n} V(\Omega)\right)^{\frac{2(l-p)}{n}}}\left(\frac{V(\Omega)}{I(\Omega)}\right)^{p} k^{\frac{2(l-p)}{n}}
\end{aligned}
$$

When $l=1$, (1.13) becomes (1.5).

In this paper, we obtain the following result for problem (1.1).

Theorem 1. Let $\Omega$ be a bounded domain in an $n$-dimensional Euclidean space $\mathbb{R}^{n}$. Denote by $\lambda_{j}$ the $j$-th eigenvalue of problem (1.1). Then we have

$$
\begin{aligned}
\frac{1}{k} \sum_{j=1}^{k} \lambda_{j} \geq & \frac{n}{n+2 l} \frac{(2 \pi)^{2 l}}{\left(\omega_{n} V(\Omega)\right)^{\frac{2 l}{n}}} k^{\frac{2 l}{n}} \\
& +\frac{n l}{48} \frac{(2 \pi)^{2 l-2}}{\left(\omega_{n} V(\Omega)\right)^{\frac{2 l-2}{n}}} \frac{V(\Omega)}{I(\Omega)} k^{\frac{2 l-2}{n}}\left(1-\varepsilon_{n}(k)\right),
\end{aligned}
$$

where $0 \leq \varepsilon_{n}(k)=O\left(k^{-\frac{2}{n}}\right)$ is a infinitesimal of $k^{-\frac{2}{n}}$.

Remark 1.1. Taking $l=1$ in (1.14), we obtain (1.6). Moreover, the second term on the right-hand side of (1.13) is

$$
\frac{l}{24(n+2 l)} \frac{(2 \pi)^{2 l-2}}{\left(\omega_{n} V(\Omega)\right)^{\frac{2 l-2}{n}}} \frac{V(\Omega)}{I(\Omega)} k^{\frac{2 l-2}{n}} .
$$

Hence, the second term on the right-hand side of (1.14) is $\frac{n(n+2 l)}{2}$ times larger than that of (1.13). Thus, for large $k,(1.14)$ is sharper than (1.13). 
Furthermore, we investigate the following Dirichlet eigenvalue problem of quadratic polynomial operator of the Laplacian:

$$
\left\{\begin{array}{l}
\Delta^{2} u-a \Delta u=\Gamma u, \quad \text { on } \Omega, \\
\left.u\right|_{\partial \Omega}=\left.\frac{\partial u}{\partial \nu}\right|_{\partial \Omega}=0
\end{array}\right.
$$

where $a$ is a nonnegative constant. Levine and Protter [8] proved that the eigenvalues of this problem satisfy

$$
\Gamma_{k} \geq \frac{n}{n+4} \frac{(2 \pi)^{4}}{\left(\omega_{n} V(\Omega)\right)^{\frac{4}{n}}} k^{\frac{4}{n}}+\frac{n a}{n+2} \frac{(2 \pi)^{2}}{\left(\omega_{n} V(\Omega)\right)^{\frac{2}{n}}} k^{\frac{2}{n}} .
$$

In this paper, we derive the following results for problem (1.15).

Theorem 2. Let $\Omega$ be a bounded domain in $\mathbb{R}^{n}$. Denote by $\Gamma_{j}$ the $j$-th eigenvalue of problem (1.15). Then we have

$$
\begin{aligned}
\frac{1}{k} \sum_{j=1}^{k} \Gamma_{j} \geq & \frac{n}{n+4} \frac{(2 \pi)^{4}}{\left(\omega_{n} V(\Omega)\right)^{\frac{4}{n}}} k^{\frac{4}{n}}+\left(\frac{n}{24} \frac{V(\Omega)}{I(\Omega)}+\frac{n a}{n+2}\right) \frac{(2 \pi)^{2}}{\left(\omega_{n} V(\Omega)\right)^{\frac{2}{n}}} k^{\frac{2}{n}} \\
& +\left[-\frac{n\left(n^{2}-4\right)}{3840} \frac{V(\Omega)}{I(\Omega)}+\frac{n a}{48}\right] \frac{V(\Omega)}{I(\Omega)}\left(1-\varepsilon_{n}(k)\right),
\end{aligned}
$$

where $0 \leq \varepsilon_{n}(k)=O\left(k^{-\frac{2}{n}}\right)$ is a infinitesimal of $k^{-\frac{2}{n}}$.

For the special cases of $n=2,3,4$, we prove the following sharper result:

Theorem 3. Denote by $\Gamma_{j}$ the $j$-th eigenvalue of problem (1.15) on a bounded domain $\Omega$ in $\mathbb{R}^{n}$, where $n=2,3,4$. Then we have

$$
\begin{aligned}
\frac{1}{k} \sum_{j=1}^{k} \Gamma_{j} \geq & \frac{n}{n+4} \frac{(2 \pi)^{4}}{\left(\omega_{n} V(\Omega)\right)^{\frac{4}{n}}} k^{\frac{4}{n}}+\left(\frac{n}{24} \alpha_{n} \frac{V(\Omega)}{I(\Omega)}+\frac{n a}{n+2}\right) \frac{(2 \pi)^{2}}{\left(\omega_{n} V(\Omega)\right)^{\frac{2}{n}}} k^{\frac{2}{n}} \\
& +\frac{n a}{48} \beta_{n} \frac{V(\Omega)}{I(\Omega)},
\end{aligned}
$$

where $\alpha_{2}=\frac{12095}{12096}, \beta_{2}=\frac{119}{120}, \alpha_{3}=0.991, \beta_{3}=0.986, \alpha_{4}=0.985$ and $\beta_{4}=0.983$.

Making a modification in the proof of Theorem 3, we can get the following result:

Theorem 4. Denote by $\Gamma_{j}$ the $j$-th eigenvalue of problem (1.15) on a bounded domain $\Omega$ in $\mathbb{R}^{n}$, where $n=3,4$. Then we have

$$
\begin{aligned}
\frac{1}{k} \sum_{j=1}^{k} \Gamma_{j} \geq & \frac{n}{n+4} \frac{(2 \pi)^{4}}{\left(\omega_{n} V(\Omega)\right)^{\frac{4}{n}}} k^{\frac{4}{n}}+\left(\frac{n}{24} \frac{V(\Omega)}{I(\Omega)}+\frac{n a}{n+2}\right) \frac{(2 \pi)^{2}}{\left(\omega_{n} V(\Omega)\right)^{\frac{2}{n}}} k^{\frac{2}{n}} \\
& +\left[-\frac{n\left(n^{2}-4\right)}{3840} \frac{V(\Omega)}{I(\Omega)}+\frac{n a}{48} \beta_{n}\right] \frac{V(\Omega)}{I(\Omega)} .
\end{aligned}
$$

Remark 1.2. Taking $a=0$ in (1.17), (1.18) and (1.19), we can get some results for the clamped plate problem. 


\section{Proofs of the main results}

In order to prove Theorem 1, we need the following lemma derived by Ilyin [6].

Lemma 1. Let

$$
\Psi_{s}(r)= \begin{cases}M, & \text { for } \quad 0 \leq r \leq s \\ M-L(r-s), & \text { for } \quad s \leq r \leq s+\frac{M}{L} \\ 0, & \text { for } \quad r \geq s+\frac{M}{L} .\end{cases}
$$

Suppose that $\int_{0}^{+\infty} r^{b} \Psi_{s}(r) d r=m^{*}$ and $d \geq b$. Then for any decreasing and absolutely continuous function $F$ satisfying the conditions

$$
0 \leq F \leq M, \quad \int_{0}^{+\infty} r^{b} F(r) d r=m^{*}, \quad 0 \leq-F^{\prime} \leq L,
$$

the following inequality holds:

$$
\int_{0}^{+\infty} r^{d} F(r) d r \geq \int_{0}^{+\infty} r^{d} \Psi_{s}(r) d r
$$

Now we give the proof of Theorem 1 .

Proof of Thereom 1 Let $u_{j}$ be an orthonormal eigenfuction corresponding to the $j$-th eigenvalue $\lambda_{j}$ of problem (1.1). Denote by $\widehat{u}_{j}(\xi)$ the Fourier transform of $u_{j}(x)$, which is defined by

$$
\widehat{u}_{j}(\xi)=(2 \pi)^{-\frac{n}{2}} \int_{\Omega} u_{j}(x) e^{i x \cdot \xi} d x
$$

It follows from Plancherel's Theorem that

$$
\int_{\Omega} \widehat{u}_{j}(\xi) \widehat{u}_{q}(\xi) d \xi=\delta_{j q}
$$

Set $h(\xi)=\sum_{j=1}^{k}\left|\widehat{u}_{j}(\xi)\right|^{2}$. From (2.4) and Bessel's inequality, one can get

$$
h(\xi)=\sum_{j=1}^{k}\left|\widehat{u}_{j}(\xi)\right|^{2} \leq(2 \pi)^{-n} \int_{\Omega}\left|e^{i x \cdot \xi}\right|^{2} d x=(2 \pi)^{-n} V(\Omega) .
$$

Moreover, Parsevel's identity implies that

$$
\int_{\mathbb{R}^{n}} h(\xi) d \xi=\sum_{j=1}^{k} \int_{\Omega}\left|u_{j}(x)\right|^{2} d x=k .
$$

Since

$$
\nabla \widehat{u}_{j}(\xi)=(2 \pi)^{-\frac{n}{2}} \int_{\Omega} i x u_{j}(x) e^{i x \cdot \xi} d x
$$


we have

$$
\sum_{j=1}^{k}\left|\nabla \widehat{u}_{j}(\xi)\right|^{2} \leq(2 \pi)^{-n} \int_{\Omega}\left|i x e^{i x \cdot \xi}\right|^{2} d x=(2 \pi)^{-n} I(\Omega) .
$$

It follows from (2.5) and (2.7) that

$$
|\nabla h(\xi)| \leq 2\left(\sum_{j=1}^{k}\left|\widehat{u}_{j}(\xi)\right|^{2}\right)^{\frac{1}{2}}\left(\sum_{j=1}^{k}\left|\nabla \widehat{u}_{j}(\xi)\right|^{2}\right)^{\frac{1}{2}} \leq 2(2 \pi)^{-n} \sqrt{V(\Omega) I(\Omega)} .
$$

Denote by $h^{*}(\xi)=\psi(|\xi|)$ the symmetric decreasing rearrangement (see [2,14]) of $h$. From

$$
k=\sum_{j=1}^{k} \int_{\Omega}\left|u_{j}(x)\right|^{2} d x=\int_{\mathbb{R}^{n}} h(\xi) d \xi=\int_{\mathbb{R}^{n}} h^{*}(\xi) d \xi=n \omega_{n} \int_{0}^{+\infty} r^{n-1} \psi(r) d r,
$$

we get

$$
\int_{0}^{+\infty} r^{n-1} \psi(r) d r=\frac{k}{n \omega_{n}}
$$

At the same time, using integration by parts and Parseval's identity, we have

$$
\begin{aligned}
\int_{\mathbb{R}^{n}}|\xi|^{2 l} h(\xi) d \xi & =\sum_{j=1}^{k} \sum_{p_{1}, \cdots, p_{l}=1}^{n} \int_{\mathbb{R}^{n}}\left|(2 \pi)^{-\frac{n}{2}} \int_{\Omega} \xi_{p_{1}} \cdots \xi_{p_{l}} u_{j}(x) e^{i x \cdot \xi} d x\right|^{2} d \xi \\
& =\sum_{j=1}^{k} \sum_{p_{1}, \cdots, p_{l}=1}^{n} \int_{\mathbb{R}^{n}}\left|(2 \pi)^{-\frac{n}{2}} \int_{\Omega} \frac{\partial^{l} u_{j}(x)}{\partial x_{p_{1}} \cdots \partial x_{p_{l}}} e^{i x \cdot \xi} d x\right|^{2} d \xi \\
& =\sum_{j=1}^{k} \sum_{p_{1}, \cdots, p_{l}=1}^{n} \int_{\mathbb{R}^{n}}\left|\frac{\partial^{l} u_{j}(\xi)}{\partial x_{p_{1}} \cdots \partial x_{p_{l}}}\right|^{2} d \xi \\
& =\sum_{j=1}^{k} \sum_{p_{1}, \cdots, p_{l}=1}^{n} \int_{\mathbb{R}^{n}}\left(\frac{\partial^{l} u_{j}(x)}{\partial x_{p_{1}} \cdots \partial x_{p_{l}}}\right)^{2} d x \\
& =\sum_{j=1}^{k} \int_{\Omega} u_{j}(x)(-\Delta)^{l} u_{j}(x) d x .
\end{aligned}
$$

Thus, it yields

$$
\sum_{j=1}^{k} \lambda_{j}=\int_{\mathbb{R}^{n}}|\xi|^{2 l} h(\xi) d \xi
$$

Making use of (2.11) and the properties of symmetric decreasing rearrangement, we obtain

$$
\sum_{j=1}^{k} \lambda_{j}=\int_{\mathbb{R}^{n}}|\xi|^{2 l} h(\xi) d \xi \geq \int_{\mathbb{R}^{n}}|\xi|^{2 l} h^{*}(\xi) d \xi=n \omega_{n} \int_{0}^{+\infty} r^{n+2 l-1} \psi(r) d r .
$$


Noticing (2.5), (2.8) and (2.9), we can apply Lemma 1 to $\psi$ with $b=n-1$ and $d=n+2 l-1$. Therefore, using (2.12), we have

$$
\sum_{j=1}^{k} \lambda_{j} \geq n \omega_{n} \int_{0}^{+\infty} r^{n+2 l-1} \psi(r) d r \geq n \omega_{n} \int_{0}^{+\infty} r^{n+2 l-1} \Psi_{s}(r) d r
$$

with $M=(2 \pi)^{-n} V(\Omega), m_{*}=\frac{k}{n \omega_{n}}$ and $L=2(2 \pi)^{-n} \sqrt{V(\Omega) I(\Omega)}$. Set $t=\frac{L s}{M}$. Combining (2.9) and

$$
\int_{0}^{+\infty} r^{n-1} \psi(r) d r=\int_{0}^{+\infty} r^{n-1} \Psi_{s}(r) d r=\frac{M^{n+1}}{n(n+1) L^{n}}\left[(t+1)^{n+1}-t^{n+1}\right],
$$

it yields

$$
(t+1)^{n+1}-t^{n+1}=k_{*}
$$

where

$$
k_{*}=k \frac{(n+1) L^{n}}{\omega_{n} M^{n+1}}
$$

Set $\eta=t-\frac{1}{2}$. Then (2.14) becomes

$$
\left(\eta+\frac{1}{2}\right)^{n+1}-\left(\eta-\frac{1}{2}\right)^{n+1}=k_{*}
$$

The asymptotic expansion for the unique positive root of (2.15) is

$$
\eta\left(k_{*}\right)=\zeta-\frac{n-1}{24} \zeta^{-1}+\frac{(n-1)(n-3)(2 n+1)}{5760} \zeta^{-3}+\cdots,
$$

where $\zeta=\left(\frac{k_{*}}{n+1}\right)^{\frac{1}{n}}$. Then we can deduce

$$
\begin{aligned}
& \left(t\left(k^{*}\right)+1\right)^{n+2 l+1}-t\left(k^{*}\right)^{n+2 l+1} \\
= & \left(\begin{array}{c}
n+2 l+1 \\
1
\end{array}\right) \zeta^{n+2 l}+2\left[\frac{1}{2^{3}}\left(\begin{array}{c}
n+2 l+1 \\
3
\end{array}\right)-\frac{n-1}{48}\left(\begin{array}{c}
n+2 l+1 \\
2
\end{array}\right)\left(\begin{array}{l}
2 \\
1
\end{array}\right)\right] \zeta^{n+2 l-2} \\
& +2\left[\frac{1}{2^{5}}\left(\begin{array}{c}
n+2 l+1 \\
5
\end{array}\right)-\frac{1}{2^{3}} \frac{n-1}{24}\left(\begin{array}{c}
n+2 l+1 \\
4
\end{array}\right)\left(\begin{array}{l}
4 \\
1
\end{array}\right)+\frac{1}{2} \frac{(n-1)^{2}}{24^{2}}\left(\begin{array}{c}
n+2 l+1 \\
3
\end{array}\right)\left(\begin{array}{l}
3 \\
1
\end{array}\right)\right. \\
& \left.+\frac{1}{2} \frac{(n-1)(n-3)(2 n+1)}{5760}\left(\begin{array}{c}
n+2 l+1 \\
2
\end{array}\right)\left(\begin{array}{l}
2 \\
1
\end{array}\right)\right] \zeta^{n+2 l-4}+\cdots \\
= & (n+2 l+1)\left[\zeta^{n+2 l}+\frac{l(n+2 l)}{12} \zeta^{n+2 l-2}+\frac{(n+2 l) C(n, l)}{5760} \zeta^{n+2 l-4}+\cdots\right],
\end{aligned}
$$

where $\left(\begin{array}{l}q \\ t\end{array}\right)=\frac{q !}{t !(q-t) !}$ and

$$
\begin{aligned}
C(n, l)= & (n+2 l-1)\left[(n+2 l-2)(6 l-7 n+1)+5(n-1)^{2}\right] \\
& +(n-1)(n-3)(2 n+1) .
\end{aligned}
$$


Using (2.17), we get

$$
\begin{aligned}
& n \omega_{n} \int_{0}^{+\infty} r^{n+2 l-1} \Psi_{s}(r) d r \\
= & \frac{n \omega_{n} M^{n+2 l+1}}{(n+2 l)(n+2 l+1) L^{n+2 l}}\left[\left(t\left(k_{*}\right)+1\right)^{n+2 l+1}-t\left(k_{*}\right)^{n+2 l+1}\right] \\
= & \frac{n \omega_{n} M^{n+2 l+1}}{(n+2 l) L^{n+2 l}}\left[\left(\frac{k_{*}}{n+1}\right)^{\frac{n+2 l}{n}}+\frac{l(n+2 l)}{12}\left(\frac{k_{*}}{n+1}\right)^{\frac{n+2 l-2}{n}}\right. \\
& \left.+\frac{(n+2 l) C(n, l)}{5760}\left(\frac{k_{*}}{n+1}\right)^{\frac{n+2 l-4}{n}}+\cdots\right] .
\end{aligned}
$$

Substituting $k_{*}=k \frac{(n+1) L^{n}}{\omega_{n} M^{n+1}}, M=(2 \pi)^{-n} V(\Omega)$ and $L=2(2 \pi)^{-n} \sqrt{V(\Omega) I(\Omega)}$ into (2.18), we have

$$
\begin{aligned}
& n \omega_{n} \int_{0}^{+\infty} r^{n+2 l-1} \Psi_{s}(r) d r \\
= & \frac{n}{n+2 l} \omega_{n}^{-\frac{2 l}{n}} M^{-\frac{2 l}{n}} k^{1+\frac{2 l}{n}}+\frac{n l}{12} \omega_{n}^{-\frac{2 l-2}{n}} \frac{M^{2-\frac{2 l-2}{n}}}{L^{2}} k^{1+\frac{2 l-2}{n}} \\
& +\frac{n C(n, l)}{5760} \omega_{n}^{-\frac{2 l-4}{n}} \frac{M^{4-\frac{2 l-4}{n}}}{L^{4}} k^{1+\frac{2 l-4}{n}}+O\left(k^{1+\frac{2 l-6}{n}}\right) \\
= & \frac{n}{n+2 l} \frac{(2 \pi)^{2 l}}{\left(\omega_{n} V(\Omega)\right)^{\frac{2 l}{n}}} k^{1+\frac{2 l}{n}}+\frac{n l}{48} \frac{(2 \pi)^{2 l-2}}{\left(\omega_{n} V(\Omega)\right)^{\frac{2 l-2}{n}}} \frac{V(\Omega)}{I(\Omega)} k^{1+\frac{2 l-2}{n}} \\
& +\frac{n C(n, l)}{92160} \frac{(2 \pi)^{2 l-4}}{\left(\omega_{n} V(\Omega)\right)^{\frac{2 l-4}{n}}}\left(\frac{V(\Omega)}{I(\Omega)}\right)^{2} k^{1+\frac{2 l-4}{n}}+O\left(k^{1+\frac{2 l-6}{n}}\right) .
\end{aligned}
$$

Inserting (2.19) into (2.13), we know that (1.14) is true. This completes the proof of Theorem 1.

Proof of Thereom 2 It follows from (2.10) that

$$
\begin{aligned}
\sum_{j=1}^{k} \Gamma_{j} & =\sum_{j=1}^{k} \int_{\Omega} u_{j}(x)\left(\Delta^{2} u_{j}(x)-a \Delta u_{j}(x)\right) d x \\
& =\int_{\mathbb{R}^{n}}|\xi|^{4} h(\xi) d \xi+a \int_{\mathbb{R}^{n}}|\xi|^{2} h(\xi) d \xi \\
& \geq \int_{\mathbb{R}^{n}}|\xi|^{4} h^{*}(\xi) d \xi+a \int_{\mathbb{R}^{n}}|\xi|^{2} h^{*}(\xi) d \xi \\
& =n \omega_{n}\left(\int_{0}^{+\infty} r^{n+3} \psi(r) d r+a \int_{0}^{+\infty} r^{n+1} \psi(r) d r\right) .
\end{aligned}
$$

Then, applying Lemma 1 to $\psi$ and using (2.20), we obtain

$$
\sum_{j=1}^{k} \Gamma_{j} \geq n \omega_{n}\left(\int_{0}^{+\infty} r^{n+3} \Psi_{s}(r) d r+a \int_{0}^{+\infty} r^{n+1} \Psi_{s}(r) d r\right) .
$$


Observe that $C(n, l)=-24 n^{2}+96$ when $l=2$ and $C(n, l)=-4(3 n+2)(n-1)$ when $l=1$. Therefore, from (2.19), we have

$$
\begin{aligned}
& n \omega_{n}\left(\int_{0}^{+\infty} r^{n+3} \Psi_{s}(r) d r+a \int_{0}^{+\infty} r^{n+1} \Psi_{s}(r) d r\right) \\
= & \frac{n}{n+4} \frac{(2 \pi)^{4}}{\left(\omega_{n} V(\Omega)\right)^{\frac{4}{n}}} k^{1+\frac{4}{n}}+\left(\frac{n}{24} \frac{V(\Omega)}{I(\Omega)}+\frac{n a}{n+2}\right) \frac{(2 \pi)^{2}}{\left(\omega_{n} V(\Omega)\right)^{\frac{2}{n}}} k^{1+\frac{2}{n}} \\
& +\left[-\frac{n\left(n^{2}-4\right)}{3840} \frac{V(\Omega)}{I(\Omega)}+a \frac{n}{48}\right] \frac{V(\Omega)}{I(\Omega)} k+O\left(k^{1-\frac{2}{n}}\right) .
\end{aligned}
$$

Then it is easy to find that (1.17) holds. This completes the proof of Theorem 2.

Proof of Thereom 3 When $n=2$, making use of (1.7) and (1.10), we have

$$
\begin{aligned}
\sum_{j=1}^{k} \Gamma_{j} & =\int_{\mathbb{R}^{n}}|\xi|^{4} h(\xi) d \xi+a \int_{\mathbb{R}^{n}}|\xi|^{2} h(\xi) d \xi \\
& \geq 2 \omega_{2} \int_{0}^{+\infty} r^{5} \Psi_{s}(r) d r+2 a \omega_{2} \int_{0}^{+\infty} r^{3} \Psi_{s}(r) d r \\
& \geq \frac{1}{3} \frac{(2 \pi)^{4}}{\left(\omega_{2} V(\Omega)\right)^{2}} k^{3}+\left(\frac{\alpha_{2}}{12 I(\Omega)}+\frac{a}{2 V(\Omega)}\right) \frac{(2 \pi)^{2}}{\omega_{2}} k^{2}+\frac{a}{24} \beta_{2} \frac{V(\Omega)}{I(\Omega)} k
\end{aligned}
$$

where $\alpha_{2}=\frac{12095}{12096}$ and $\beta_{2}=\frac{119}{120}$.

When $n=3$, it follows from (2.21) that

$$
\sum_{j=1}^{k} \Gamma_{j} \geq 3 \omega_{3} \int_{0}^{+\infty} r^{6} \Psi_{s}(r) d r+3 a \omega_{3} \int_{0}^{+\infty} r^{4} \Psi_{s}(r) d r
$$

Now we make an estimate for the lower bound of $\int_{0}^{+\infty} r^{6} \Psi_{s}(r) d r$. Since

$$
\int_{0}^{+\infty} r^{6} \Psi_{s}(r) d r=\frac{M^{8}}{56 L^{7}}\left[\left(t\left(k_{*}\right)+1\right)^{8}-t\left(k_{*}\right)^{8}\right]
$$

we need to estimate $\left(t\left(k_{*}\right)+1\right)^{8}-t\left(k_{*}\right)^{8}$. The equation $(2.14)$ becomes $(t+1)^{4}-t^{4}=$ $k_{*}$ when $n=3$. Its positive root $t\left(k_{*}\right)$ is

$$
t\left(k_{*}\right)=\frac{1}{2}\left(\rho\left(k_{*}\right)-\varrho\left(k_{*}\right)\right)-\frac{1}{2},
$$

where $\rho\left(k_{*}\right)=\left(k_{*}+\sqrt{k_{*}^{2}+\frac{1}{27}}\right)^{\frac{1}{3}}$ and $\varrho\left(k_{*}\right)=\left(-k_{*}+\sqrt{k_{*}^{2}+\frac{1}{27}}\right)^{\frac{1}{3}}$. Set $\vartheta\left(k_{*}\right)=$ $\frac{1}{2}\left(\rho\left(k_{*}\right)-\varrho\left(k_{*}\right)\right)$. Then we have

$$
\begin{aligned}
\left(t\left(k_{*}\right)+1\right)^{8}-t\left(k_{*}\right)^{8}= & 8 \vartheta\left(k_{*}\right)^{7}+14 \vartheta\left(k_{*}\right)^{5}+\frac{7}{2} \vartheta\left(k_{*}\right)^{3}+\frac{1}{8} \vartheta\left(k_{*}\right) \\
=\frac{1}{16} & {\left[\left(\rho\left(k_{*}\right)-\varrho\left(k_{*}\right)\right)^{7}+7\left(\rho\left(k_{*}\right)-\varrho\left(k_{*}\right)\right)^{5}\right.} \\
& \left.+7\left(\rho\left(k_{*}\right)-\varrho\left(k_{*}\right)\right)^{3}+\left(\rho\left(k_{*}\right)-\varrho\left(k_{*}\right)\right)\right] .
\end{aligned}
$$


Observe that

$$
\rho\left(k_{*}\right) \cdot \varrho\left(k_{*}\right)=\frac{1}{3} .
$$

Then, using (2.26), we have

$$
\begin{aligned}
& \left(\rho\left(k_{*}\right)-\varrho\left(k_{*}\right)\right)^{7} \\
= & \rho\left(k_{*}\right)\left(\rho\left(k_{*}\right)^{6}+7 \varrho\left(k_{*}\right)^{6}\right)+21 \rho\left(k_{*}\right)^{2} \varrho\left(k_{*}\right)^{2}\left(\rho\left(k_{*}\right)^{3}-\varrho\left(k_{*}\right)^{3}\right) \\
& -35 \rho\left(k_{*}\right)^{3} \varrho\left(k_{*}\right)^{3}\left(\rho\left(k_{*}\right)-\varrho\left(k_{*}\right)\right)-\varrho\left(k_{*}\right)\left(7 \rho\left(k_{*}\right)^{6}+\varrho\left(k_{*}\right)^{6}\right) \\
= & \left(k_{*}+\sqrt{k_{*}^{2}+\frac{1}{27}}\right)^{\frac{1}{3}}\left(16 k_{*}^{2}-12 k_{*} \sqrt{k_{*}^{2}+\frac{1}{27}}-1\right)+\frac{14}{3} k_{*} \\
& -\left(-k_{*}+\sqrt{k_{*}^{2}+\frac{1}{27}}\right)^{\frac{1}{3}}\left(16 k_{*}^{2}+12 k_{*} \sqrt{k_{*}^{2}+\frac{1}{27}}-1\right), \\
\left(\rho\left(k_{*}\right)-\varrho\left(k_{*}\right)\right)^{5} & \rho\left(k_{*}\right)^{5}-5 \rho\left(k_{*}\right) \varrho\left(k_{*}\right)\left(\rho\left(k_{*}\right)^{3}-\varrho\left(k_{*}\right)^{3}\right)+10 \rho\left(k_{*}\right)^{2} \varrho\left(k_{*}\right)^{2}\left(\rho\left(k_{*}\right)-\varrho\left(k_{*}\right)\right)-\varrho\left(k_{*}\right)^{5} \\
=\left(k_{*}+\right. & \left.\sqrt{k_{*}^{2}+\frac{1}{27}}\right)^{\frac{5}{3}}-\left(-k_{*}+\sqrt{k_{*}^{2}+\frac{1}{27}}\right)^{\frac{5}{3}}-\frac{10}{3} k_{*}+\frac{10}{9}\left(k_{*}+\sqrt{k_{*}^{2}+\frac{1}{27}}\right)^{\frac{1}{3}} \\
-\frac{10}{9} & \left(-k_{*}+\sqrt{k_{*}^{2}+\frac{1}{27}}\right)^{\frac{1}{3}}
\end{aligned}
$$

and

$$
\begin{aligned}
& 7\left(\rho\left(k_{*}\right)-\varrho\left(k_{*}\right)\right)^{3}+\left(\rho\left(k_{*}\right)-\varrho\left(k_{*}\right)\right) \\
= & 7\left[\rho\left(k_{*}\right)^{3}-\varrho\left(k_{*}\right)^{3}-3 \rho\left(k_{*}\right) \varrho\left(k_{*}\right)\left(\rho\left(k_{*}\right)-\varrho\left(k_{*}\right)\right)\right]+\left(\rho\left(k_{*}\right)-\varrho\left(k_{*}\right)\right) \\
= & 14 k_{*}-6\left(k_{*}+\sqrt{k_{*}^{2}+\frac{1}{27}}\right)^{\frac{1}{3}}+6\left(-k_{*}+\sqrt{k_{*}^{2}+\frac{1}{27}}\right)^{\frac{1}{3}} .
\end{aligned}
$$

Substituting (2.27-2.29) into (2.25), we obtain

$$
\begin{aligned}
& \left(t\left(k_{*}\right)+1\right)^{8}-t\left(k_{*}\right)^{8} \\
= & \left(k_{*}+\sqrt{k_{*}^{2}+\frac{1}{27}}\right)^{\frac{1}{3}}\left(k_{*}^{2}-\frac{3}{4} k_{*} \sqrt{k_{*}^{2}+\frac{1}{27}}\right)-\frac{7}{16}\left(-k_{*}+\sqrt{k_{*}^{2}+\frac{1}{27}}\right)^{\frac{5}{3}} \\
& +\left[\frac{7}{16}\left(k_{*}+\sqrt{k_{*}^{2}+\frac{1}{27}}\right)^{\frac{5}{3}}-\left(-k_{*}+\sqrt{k_{*}^{2}+\frac{1}{27}}\right)^{\frac{1}{3}}\left(k_{*}^{2}+\frac{3}{4} k_{*} \sqrt{k_{*}^{2}+\frac{1}{27}}\right)\right] \\
& +\frac{7}{144}\left[\left(k_{*}+\sqrt{k_{*}^{2}+\frac{1}{27}}\right)^{\frac{1}{3}}-\left(-k_{*}+\sqrt{k_{*}^{2}+\frac{1}{27}}\right)^{\frac{1}{3}}\right]-\frac{7}{24} k_{*} .
\end{aligned}
$$

Now we make some estimates for some terms in the right hand side of (2.30). The first term can be estimated as follows:

$$
\left(k_{*}+\sqrt{k_{*}^{2}+\frac{1}{27}}\right)^{\frac{1}{3}}\left(k_{*}^{2}-\frac{3}{4} k_{*} \sqrt{k_{*}^{2}+\frac{1}{27}}\right) \geq \frac{2^{\frac{1}{3}}}{4} k_{*}^{\frac{7}{3}}-\frac{2^{\frac{1}{3}}}{72} k_{*}^{\frac{1}{3}} .
$$


Here we use the inequality $\sqrt{k_{*}^{2}+\frac{1}{27}} \leq k_{*}+\frac{1}{54 k_{*}}$ since $k_{*}$ is large. The second term is

$$
-\frac{7}{16}\left(-k_{*}+\sqrt{k_{*}^{2}+\frac{1}{27}}\right)^{\frac{5}{3}} \geq-\frac{7 \cdot 2^{\frac{1}{3}}}{16 \cdot 54 \cdot 18} k_{*}^{-\frac{5}{3}} .
$$

The third term is

$$
\begin{aligned}
& \frac{7}{16}\left(k_{*}+\sqrt{k_{*}^{2}+\frac{1}{27}}\right)^{\frac{5}{3}}-\left(-k_{*}+\sqrt{k_{*}^{2}+\frac{1}{27}}\right)^{\frac{1}{3}}\left(k_{*}^{2}+\frac{3}{4} k_{*} \sqrt{k_{*}^{2}+\frac{1}{27}}\right) \\
\geq & \frac{7 \cdot 2^{\frac{2}{3}}}{12} k_{*}^{\frac{5}{3}}-\frac{2^{\frac{2}{3}}}{432} k_{*}^{-\frac{1}{3}} .
\end{aligned}
$$

The fourth term is

$$
\frac{7}{144}\left[\left(k_{*}+\sqrt{k_{*}^{2}+\frac{1}{27}}\right)^{\frac{1}{3}}-\left(-k_{*}+\sqrt{k_{*}^{2}+\frac{1}{27}}\right)^{\frac{1}{3}}\right] \geq \frac{7 \cdot 2^{\frac{1}{3}}}{144} k_{*}^{\frac{1}{3}}-\frac{7 \cdot 2^{\frac{2}{3}}}{16 \cdot 54} k_{*}^{-\frac{1}{3}} .
$$

Therefore, using (2.31-2.34) in (2.30), we have

$$
\begin{aligned}
\left(t\left(k_{*}\right)+1\right)^{8}-t\left(k_{*}\right)^{8} \geq & \frac{2^{\frac{1}{3}}}{4} k_{*}^{\frac{7}{3}}+\frac{7 \cdot 2^{\frac{2}{3}}}{12} k_{*}^{\frac{5}{3}}-\frac{7}{24} k_{*}+\frac{5 \cdot 2^{\frac{1}{3}}}{144} k_{*}^{\frac{1}{3}}-\frac{2^{\frac{2}{3}}}{96} k_{*}^{-\frac{1}{3}} \\
& -\frac{7 \cdot 2^{\frac{1}{3}}}{16 \cdot 54 \cdot 18} k_{*}^{-\frac{5}{3}} \\
\geq & \frac{2^{\frac{1}{3}}}{4} k_{*}^{\frac{7}{3}}+\frac{7 \cdot 2^{\frac{2}{3}}}{12} k_{*}^{\frac{5}{3}}-\frac{7}{24} k_{*} .
\end{aligned}
$$

Here we used the fact that $k_{*} \geq 1$. In fact, noticing $k_{*} \geq \frac{(n+1)(4 \pi)^{n}}{\omega_{n}^{2}}\left(\frac{n}{n+2}\right)^{\frac{n}{2}}$, it is not difficult to observe that $k_{*}=4 k L^{3}\left(\omega_{3}\right)^{-1} M^{-4} \geq \tau:=\frac{432 \sqrt{15} \pi}{25} \approx 210.25$ when $n=3$. Hence, when $\alpha \geq \frac{7}{24} \tau^{-\frac{2}{3}}$, the following inequality

$$
\alpha k_{*}^{\frac{5}{3}} \geq \frac{7}{24} k_{*}
$$

holds for $k_{*} \in[\tau,+\infty)$. Since $1-\frac{6}{7} \cdot 2^{\frac{1}{3}} \alpha \leq 1-\frac{1}{4} \cdot 2^{\frac{1}{3}} \tau^{-\frac{2}{3}} \approx 0.9911$, we can conclude that

$$
\left(t\left(k_{*}\right)+1\right)^{8}-t\left(k_{*}\right)^{8} \geq \frac{2^{\frac{1}{3}}}{4} k_{*}^{\frac{7}{3}}+\frac{7 \cdot 2^{\frac{2}{3}}}{12} \alpha_{3} k_{*}^{\frac{5}{3}}
$$

where $\alpha_{3}=0.991$. Therefore, using (2.36), we derive

$$
\begin{aligned}
3 \omega_{3} \int_{0}^{+\infty} r^{6} \Psi_{s}(r) d r & =\frac{3 \omega_{3} M^{8}}{56 L^{7}}\left[\left(t\left(k_{*}\right)+1\right)^{8}-t\left(k_{*}\right)^{8}\right] \\
& \geq \frac{3 \cdot 2^{\frac{1}{3}} \omega_{3} M^{8}}{224 L^{7}} k_{*}^{\frac{7}{3}}+\frac{2^{\frac{2}{3}} \omega_{3} M^{8}}{32 L^{7}} \alpha_{3} k_{*}^{\frac{5}{3}} \\
& =\frac{3}{7} \frac{(2 \pi)^{4}}{\left(\omega_{3} V(\Omega)\right)^{\frac{4}{3}}} k^{\frac{7}{3}}+\frac{1}{8} \alpha_{3} \frac{(2 \pi)^{2}}{\left(\omega_{3} V(\Omega)\right)^{\frac{2}{3}}} \frac{V(\Omega)}{I(\Omega)} k^{\frac{5}{3}} .
\end{aligned}
$$


At the same time, it follows from (1.7) that

$$
3 \omega_{3} \int_{0}^{+\infty} r^{4} \Psi_{s}(r) d r \geq \frac{3}{5} \frac{(2 \pi)^{2}}{\left(\omega_{3} V(\Omega)\right)^{\frac{2}{3}}} k^{\frac{5}{3}}+\frac{1}{16} \beta_{3} \frac{V(\Omega)}{I(\Omega)} k,
$$

where $\beta_{3}=0.986$. Substituting (2.37) and (2.38) into (2.24), we obtain

$$
\begin{aligned}
\sum_{j=1}^{k} \Gamma_{j} \geq & \frac{3}{7} \frac{(2 \pi)^{4}}{\left(\omega_{3} V(\Omega)\right)^{\frac{4}{3}}} k^{\frac{7}{3}}+\left(\frac{1}{8} \alpha_{3} \frac{V(\Omega)}{I(\Omega)}+\frac{3 a}{5}\right) \frac{(2 \pi)^{2}}{\left(\omega_{3} V(\Omega)\right)^{\frac{2}{3}}} k^{\frac{5}{3}} \\
& +\frac{a}{16} \beta_{3} \frac{V(\Omega)}{I(\Omega)} k .
\end{aligned}
$$

When $n=4$, it follows from (2.21) that

$$
\sum_{j=1}^{k} \Gamma_{j} \geq 4 \omega_{4} \int_{0}^{+\infty} r^{7} \Psi_{s}(r) d r+4 a \omega_{4} \int_{0}^{+\infty} r^{5} \Psi_{s}(r) d r
$$

Now we make an estimate for the lower bound of $\int_{0}^{+\infty} r^{7} \Psi_{s}(r) d r$. Since

$$
\int_{0}^{+\infty} r^{7} \Psi_{s}(r) d r=\frac{M^{9}}{72 L^{8}}\left[\left(t\left(k_{*}\right)+1\right)^{9}-t\left(k_{*}\right)^{9}\right],
$$

we need to estimate $\left(t\left(k_{*}\right)+1\right)^{9}-t\left(k_{*}\right)^{9}$. The equation $(2.14)$ becomes $(t+1)^{5}-t^{5}=$ $k_{*}$ when $n=4$. Its positive root $t\left(k_{*}\right)$ is

$$
t\left(k_{*}\right)=\theta\left(k_{*}\right)-\frac{1}{2}
$$

where $\theta\left(k_{*}\right)=\sqrt{\frac{\sqrt{20 k_{*}+5}}{10}-\frac{1}{4}}$. Then we have

$$
\begin{aligned}
& \left(t\left(k_{*}\right)+1\right)^{9}-t\left(k_{*}\right)^{9} \\
= & 9 \theta\left(k_{*}\right)^{8}+21 \theta\left(k_{*}\right)^{6}+\frac{63}{8} \theta\left(k_{*}\right)^{4}+\frac{9}{16} \theta\left(k_{*}\right)^{2}+\frac{1}{2^{8}} \\
= & \frac{9}{25} k_{*}^{2}+\frac{6}{25} k_{*} \sqrt{20 k_{*}+5}-\frac{18}{25} k_{*}+\frac{3}{50} \sqrt{20 k_{*}+5}-\frac{7}{50} \\
\geq & \frac{9}{25} k_{*}^{2}+\frac{12 \sqrt{5}}{25} k_{*}^{\frac{3}{2}}-\frac{18}{25} k_{*} .
\end{aligned}
$$

Here we used the fact that $k_{*} \geq 1$. In fact, noticing $k_{*} \geq \frac{(n+1)(4 \pi)^{n}}{\omega_{n}^{2}}\left(\frac{n}{n+2}\right)^{\frac{n}{2}}$, it is not difficult to observe that $k_{*}=5 k L^{4}\left(\omega_{4}\right)^{-1} M^{-5} \geq \sigma:=\frac{5 \cdot 2^{12}}{9} \approx 2275.56$ when $n=4$. Hence, when $\alpha \geq \frac{18}{25} \sigma^{-\frac{1}{2}}$, the following inequality

$$
\alpha k_{*}^{\frac{3}{2}} \geq \frac{18}{25} k_{*}
$$


holds for $k_{*} \in[\sigma,+\infty)$. Since $1-\frac{5 \sqrt{5}}{12} \alpha \leq 1-\frac{3 \sqrt{5}}{10} \sigma^{-\frac{1}{2}} \approx 0.9859$, we can conclude that

$$
\left(t\left(k_{*}\right)+1\right)^{9}-t\left(k_{*}\right)^{9} \geq \frac{9}{25} k_{*}^{2}+\frac{12 \sqrt{5}}{25} \alpha_{4} k_{*}^{\frac{3}{2}},
$$

where $\alpha_{4}=0.985$. Therefore, using (2.42), we deduce

$$
\begin{aligned}
4 \omega_{4} \int_{0}^{+\infty} r^{7} \Psi_{s}(r) d r & =\frac{\omega_{4} M^{9}}{18 L^{8}}\left[\left(t\left(k_{*}\right)+1\right)^{9}-t\left(k_{*}\right)^{9}\right] \\
& \geq \frac{\omega_{4} M^{9}}{50 L^{8}} k_{*}^{2}+\frac{2 \sqrt{5} \omega_{4} M^{9}}{75 L^{8}} \alpha_{4} k_{*}^{\frac{3}{2}} \\
& =\frac{1}{2} \frac{(2 \pi)^{4}}{\omega_{4} V(\Omega)} k^{2}+\frac{1}{6} \alpha_{4} \frac{(2 \pi)^{2}}{\left(\omega_{4} V(\Omega)\right)^{\frac{1}{2}}} \frac{V(\Omega)}{I(\Omega)} k^{\frac{3}{2}}
\end{aligned}
$$

Meanwhile, from (1.7), we have

$$
4 \omega_{4} \int_{0}^{+\infty} r^{5} \Psi_{s}(r) d r \geq \frac{2}{3} \frac{(2 \pi)^{2}}{\left(\omega_{4} V(\Omega)\right)^{\frac{1}{2}}} k^{\frac{3}{2}}+\frac{1}{12} \beta_{4} \frac{V(\Omega)}{I(\Omega)} k,
$$

where $\beta_{4}=0.983$. Substituting (2.43) and (2.44) into (2.40), we obtain

$$
\begin{aligned}
\sum_{j=1}^{k} \Gamma_{j} \geq & \frac{1}{2} \frac{(2 \pi)^{4}}{\omega_{4} V(\Omega)} k^{2}+\left(\frac{1}{6} \alpha_{4} \frac{V(\Omega)}{I(\Omega)}+\frac{2 a}{3}\right) \frac{(2 \pi)^{2}}{\left(\omega_{4} V(\Omega)\right)^{\frac{1}{2}}} k^{\frac{3}{2}} \\
& +\frac{a}{12} \beta_{4} \frac{V(\Omega)}{I(\Omega)} k .
\end{aligned}
$$

Therefore, synthesizing (2.23), (2.39) and (2.45), we conclude that (1.18) is true. This concludes the proof of Theorem 3 .

Proof of Thereom 4 When $n=3$, using (2.35), we derive

$$
\begin{aligned}
3 \omega_{3} \int_{0}^{+\infty} r^{6} \Psi_{s}(r) d r & \geq \frac{3 \cdot 2^{\frac{1}{3}} \omega_{3} M^{8}}{224 L^{7}} k_{*}^{\frac{7}{3}}+\frac{2^{\frac{2}{3}} \omega_{3} M^{8}}{32 L^{7}} k_{*}^{\frac{5}{3}}-\frac{\omega_{3} M^{8}}{64 L^{7}} k_{*} \\
& =\frac{3}{7} \frac{(2 \pi)^{4}}{\left(\omega_{3} V(\Omega)\right)^{\frac{4}{3}}} k^{\frac{7}{3}}+\frac{1}{8} \frac{(2 \pi)^{2}}{\left(\omega_{3} V(\Omega)\right)^{\frac{2}{3}}} \frac{V(\Omega)}{I(\Omega)} k^{\frac{5}{3}}-\frac{1}{256}\left(\frac{V(\Omega)}{I(\Omega)}\right)^{2} k
\end{aligned}
$$

Substituting (2.38) and (2.46) into (2.24), we have

$$
\begin{aligned}
\sum_{j=1}^{k} \Gamma_{j} \geq & \frac{3}{7} \frac{(2 \pi)^{4}}{\left(\omega_{3} V(\Omega)\right)^{\frac{4}{3}}} k^{\frac{7}{3}}+\left(\frac{1}{8} \frac{V(\Omega)}{I(\Omega)}+\frac{3 a}{5}\right) \frac{(2 \pi)^{2}}{\left(\omega_{3} V(\Omega)\right)^{\frac{2}{3}}} k^{\frac{5}{3}} \\
& +\left(-\frac{1}{256} \frac{V(\Omega)}{I(\Omega)}+\frac{a}{16} \beta_{3}\right) \frac{V(\Omega)}{I(\Omega)} k
\end{aligned}
$$


When $n=4$, it follows from (2.41) that

$$
\begin{aligned}
4 \omega_{4} \int_{0}^{+\infty} r^{7} \Psi_{s}(r) d r & \geq \frac{\omega_{4} M^{9}}{50 L^{8}} k_{*}^{2}+\frac{2 \sqrt{5} \omega_{4} M^{9}}{75 L^{8}} k_{*}^{\frac{3}{2}}-\frac{\omega_{4} M^{9}}{25 L^{8}} k_{*} \\
& =\frac{1}{2} \frac{(2 \pi)^{4}}{\omega_{4} V(\Omega)} k^{2}+\frac{1}{6} \frac{(2 \pi)^{2}}{\left(\omega_{4} V(\Omega)\right)^{\frac{1}{2}}} \frac{V(\Omega)}{I(\Omega)} k^{\frac{3}{2}}-\frac{1}{80}\left(\frac{V(\Omega)}{I(\Omega)}\right)^{2} k .
\end{aligned}
$$

Substituting (2.44) and (2.48) into (2.40), we obtain

$$
\begin{aligned}
\sum_{j=1}^{k} \Gamma_{j} \geq & \frac{1}{2} \frac{(2 \pi)^{4}}{\omega_{4} V(\Omega)} k^{2}+\left(\frac{1}{6} \frac{V(\Omega)}{I(\Omega)}+\frac{2 a}{3}\right) \frac{(2 \pi)^{2}}{\left(\omega_{4} V(\Omega)\right)^{\frac{1}{2}}} k^{\frac{3}{2}} \\
& +\left(-\frac{1}{80} \frac{V(\Omega)}{I(\Omega)}+\frac{a}{12} \beta_{4}\right) \frac{V(\Omega)}{I(\Omega)} k
\end{aligned}
$$

Therefore, combining (2.47) and (2.49), we conclude that (1.19) is true. This completes the proof of Theorem 4 .

\section{References}

[1] S. Agmon, On kernels, eigenvalues and eigenfunctions of operators related to elliptic problems, Comm. Pure Appl. Math. 18 (1965), 627-663.

[2] C. Bandle, Isoperimetric inequalities and applications, Pitman Monographs and Studies in Mathematics, vol. 7, Pitman, Boston, 1980.

[3] F. A. Berezin, Covariant and contravariant symbols of operators, Izv. Akad. Nauk SSSR Ser. Mat. 37 (1972), 1134-1167.

[4] Q. -M. Cheng, X. R. Qi and G. X. Wei, A lower bound for eigenvalues of the poly-Laplacian with arbitrary order, arXiv:1012.3006v1.

[5] Q. -M. Cheng and G. X. Wei, A lower bound for eigenvalues of a clamped plate problem, Calc. Var. 42 (2011), 579-590.

[6] A. A. Ilyin, Lower bounds for the spectrum of the Laplacian and Stokes operators, Discrete Cont. Dyn. S. 28 (2010), 131-146.

[7] A. Laptev and T. Weidl, Recent results on Lieb-Thirring inequalities, Journées Équations aux Dérivées Partielles (La Chapelle sur Erdre, 2000), Exp. No. XX, 14 pp., Univ. Nantes, Nantes, 2000 .

[8] H. A. Levine and M. H. Protter, Unrestricted lower bounds for eigenvalues for classes of elliptic equations and systems of equations with applications to problems in elasticity, Math. Methods Appl. Sci. 7(2) (1985), 210-222.

[9] P. Li and S. T. Yau, On the Schrödinger equations and the eigenvalue problem, Comm. Math. Phys. 88 (1983), 309-318.

[10] E. Lieb, The number of bound states of one-body Schröinger operators and the Weyl problem, Proc. Symp. Pure Math. 36 (1980), 241-252. 
[11] A. D. Melas, A lower bound for sums of eigenvalues of the Laplacian, Proc. Amer. Math. Soc. 131 (2003), 631-636.

[12] A. Pleijel, On the eigenvalues and eigenfunctions of elastic plates, Comm. Pure Appl. Math. 3 (1950), 1-10.

[13] G. Pólya, On the eigenvalues of vibrating membranes, Proc. Lond. Math. Soc. 11 (1961), 419-433.

[14] G. Pólya and G. Szegö, Isoperimetric inequalities in mathematical physics, Annals of mathematics studies, number 27, Princeton university press, Princeton, New Jersey, 1951.

QING-Ming Cheng

Department of Mathematics, Graduate School of Science and Engineering,

SAGA UNIVERSITY, SAGA 840-8502, JAPAN

E-mail address: cheng@ms.saga-u.ac.jp

He-Jun Sun

Department of Applied Mathematics, College of Science, Nanjing

University of Science And Technology, Nanjing 210094, P. R. China

E-mail address: hejunsun@163.com

Guoxin WeI

School of Mathematical Sciences, South China Normal University, Guangzhou 510631, P. R. China

E-mail address: weigx03@mails.tsinghua.edu.cn

LiNGZHONG ZENG

Department of Mathematics, Graduate School of Science and Engineering, SAga University, SAGa 840-8502, JAPAN

E-mail address: lingzhongzeng@yeah.net 